\title{
Antibiotics Susceptibility of Some Uropathogenic Bacterial Isolates from Ahmadu Bello University Teaching Hospital Zaria, Nigeria
}

\author{
Maimuna Soba Haruna ${ }^{1}$, Joshua Magu ${ }^{2}$, Joy Idume ${ }^{2}$ and Nosiri $C^{3}$, M.A. Garba \\ ${ }^{I}$ (Department of Medical Lab Science, Shehu Idris College of Health Sciences and Technology, Kaduna, \\ Nigeria) \\ ${ }_{2}^{2}$ (Department of Medical Lab Science, Shehu Idris College of Health Sciences and Technology, Kaduna, \\ Nigeria) \\ ${ }^{3}$ (Department of Pharmacy, Shehu Idris College of Health Sciences and Technology, Kaduna State, Nigeria) \\ Department of Pharmacy, Shehu Idris College of Health Sciences and Technology, Kaduna State Nigeria
}

\begin{abstract}
Urinary tract infection is an infection anywhere in the urinary tract. 300 samples of urine were collected from male and female patients of different age groups with symptoms of Urinary Tract Infection attending Ahmadu Bello University Teaching Hospital Zaria, Nigeria. The samples were screened for bacteriuria using bacteriological methods. Five types of uropathogens were identified namely Escherichia coli (E.coli), Proteus Species, Pseudomonas species, Klebsiella and Staphylococcus aureus. Urine samples of 181 females examined showed that 51 (28.2\%) were infected and from 119 males, 31 (26.1\%) were infected. E. coli had the highest occurence seen in 42 (51\%) of 82 (27. 3\%) infected cases. Out of this 42 samples with E.coli, 25 (59.5\%) were from females. E. coli was highly sensitive to Nitrofurantion (97.6\%), and highly resistant to cotrimoxazole(76.1\%). This study shows that the commonest microorganism causing UTI in this community is E. coli and drug of choice is Nitrofurantoin.
\end{abstract}

Keywords: Antimicrobial agents, bacteriuria, E.coli, Patients, Urinary Tract Infection

\section{Introduction}

Urinary tract infection is an infection anywhere in the urinary tract. (Feld and Mattoo, 2010). Urinary tract infection (UTI) is the most common infection experienced by humans after respiratory and gastro-intestinal infections, and also the most common cause of both community-acquired and nosocomial infections for patients admitted to hospitals. (Najar et al, 2009). UTI can be asymptomatic or symptomatic characterized by a wide range of symptoms from mild irritation voiding to bacteremia, sepsis or even death (Ranjbar et al, 2009)The lining of the bladder is relatively resistant to infection by most microorganisms as long as normal flow of liquid through the urinary tract is maintained (Richard, 1983).

Urinary tract infection occurs more frequently in females than males due to the shortness and width of the female urethra (Inabo and Obinibi, 2006). The incidence is a major cause for concern as it affects females of all ages as from ages when they start going to school from age of five (John, 1976). Most bacteria found in the urinary tract emanate from the intestine (Ozumba, 1993). Urinary tract infection involves bacterial invasion of any part of the urinary tract be it the kidney, ureter, bladder and the urethra (Scully, 1990). Symptomatic urinary tract infection involving the bladder known as cystitis is characterized by pain when passing out urine, frequency of urine passage and urgency coupled occasionally with the feeling that the bladder is not completely emptied (Nicolle, 2005).

An antibiotic is any microbial product which even at very low concentration inhibits or kills certain microorganisms. However, no antibiotic is effective against all bacteria (Paul, 1997). The extensive use and misuse of antimicrobial drugs such as wrong prescription, dosing, dosage, self medication as they are easily available over the counter, fake and substandard quality and poor hygienic environment have favored the increased emergence and survival of resistant strains of microorganisms which is now making it difficult to treat some infectious diseases. Antibiotic sensitivity of bacteria depends to a large extent on the pattern of use of antibiotic in a particular environment. It varies from one geographical environment to the other and over years (Gruneberg, 1980., Saffar et al., 2008). Owing to the prevalence of gastrointestinal, respiratory and other infections in the tropics, the same antibiotics are used for urinary tract infection. This study therefore aims to investigate the pathogens isolated from patients with UTI and evaluate their in-vitro susceptibility pattern to commonly used antibiotics in this community, Zaria Nigeria. 


\section{Materials And Methods}

Three hundred samples of urine from Male and Female patients of different age groups excluding pregnant women attending Ahmadu Bello University Teaching hospital, Zaria, Kaduna State were collected in sterile universal bottles and studied. Samples that showed significant bacteriuria were studied and isolates were identified using bacteriological methods. Test for antibiotic sensitivity was done according to the National Committee for Clinical Laboratory standards by using the disc diffusion method. Interpretations as "sensitive" or "resistant" was performed on the basis of diameters of zones of inhibition of the growth of bacteria according to disc manufacturer's instruction. Sensitivity was shown by zone of inhibition equal, wider or not more than $3 \mathrm{~mm}$ smaller than those of control. Resistance was shown by no zone of inhibition or zone radius measuring only $2 \mathrm{~mm}$ or less.

The micro organisms were tested for antimicrobial susceptibility against ten (10) antimicrobial agents. Gram positive and gram negative multi discs were used. The antibiotics include Ampicillin, Gentamycin, Colistin sulphate, Streptomycin,Tetracycline, Clotrimazole, Cloxacillin, Nalidixic acid, Nitrofurantoin and Ofloxacin.

\section{Data Analysis}

Results were analysed statistically using the Statistical Program for Social Sciences (SPSS) version 17.

\section{Results}

The results of the tests done are shown below.

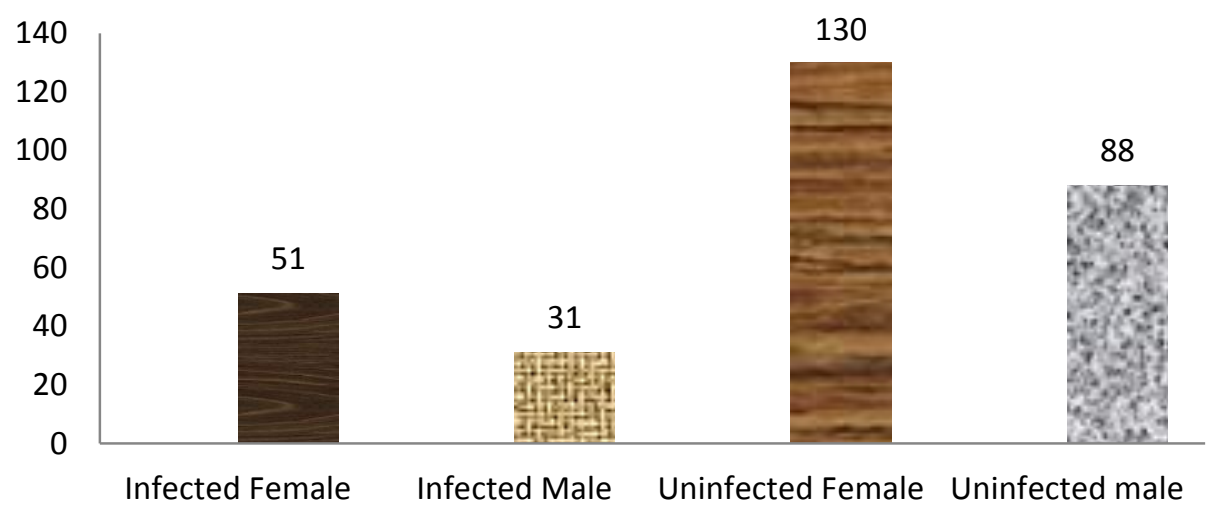

Fig.1 above shows the number of infected and uninfected patients. Out of the total number of 300 samples screened, 119 (39.6\%) were from male and 181 (60.3\%) were from female patients. Of this 181 female urine examined, $51(28.2 \%)$ were infected while $130(71.8 \%)$ were uninfected. Out of 119 male urine examined, $31(26.1 \%)$ were infected while $88(73.9 \%)$ were uninfected. A total of 82 $(27.3 \%)$ urine samples from both male and female patients were infected whereas $218(72.7 \%)$ of them were uninfected.

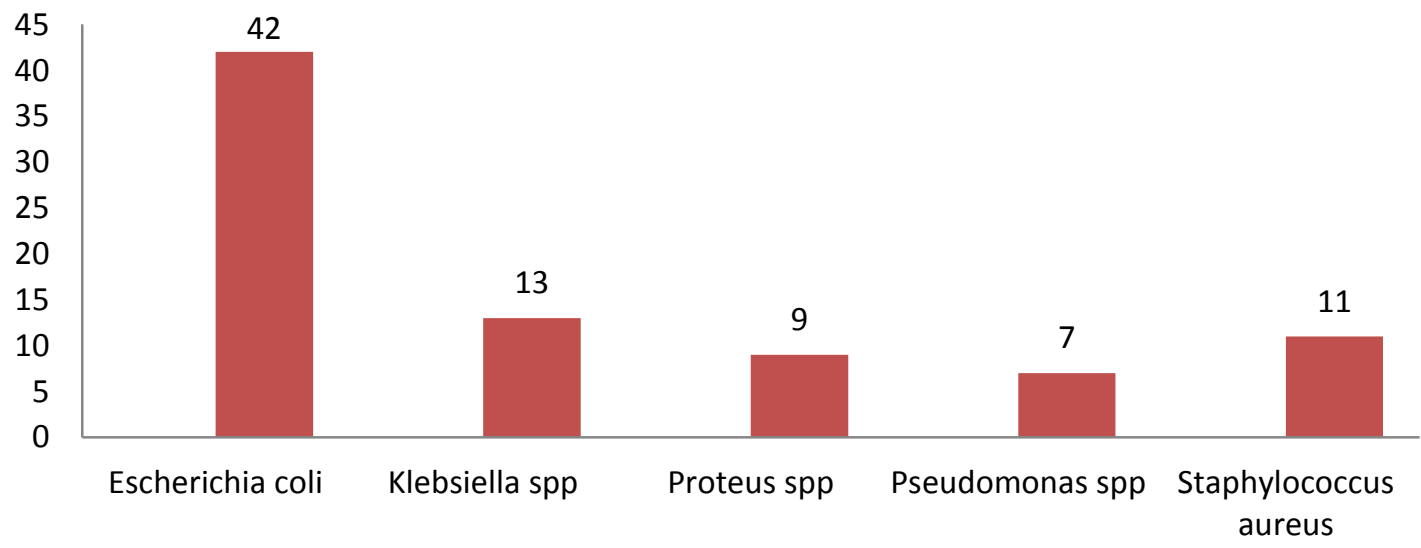

Fig. 2 above shows the different urinary pathogens isolated and their percentages. Escherichia coli had the highest incidence, occurring in $42(51.2 \%)$ out of 82 infected cases, followed by Klebsiella spp. occurring in 13 
$(15.9 \%)$ cases, and Staphylococcus aureus occurring in $11(13.4 \%)$ cases. The three pathogens accounted for $(80.5 \%)$ of the infection. Proteus spp. and Pseudomonas spp. occurred in $9(11.0 \%)$ and $7(8.5 \%)$ cases respectively.

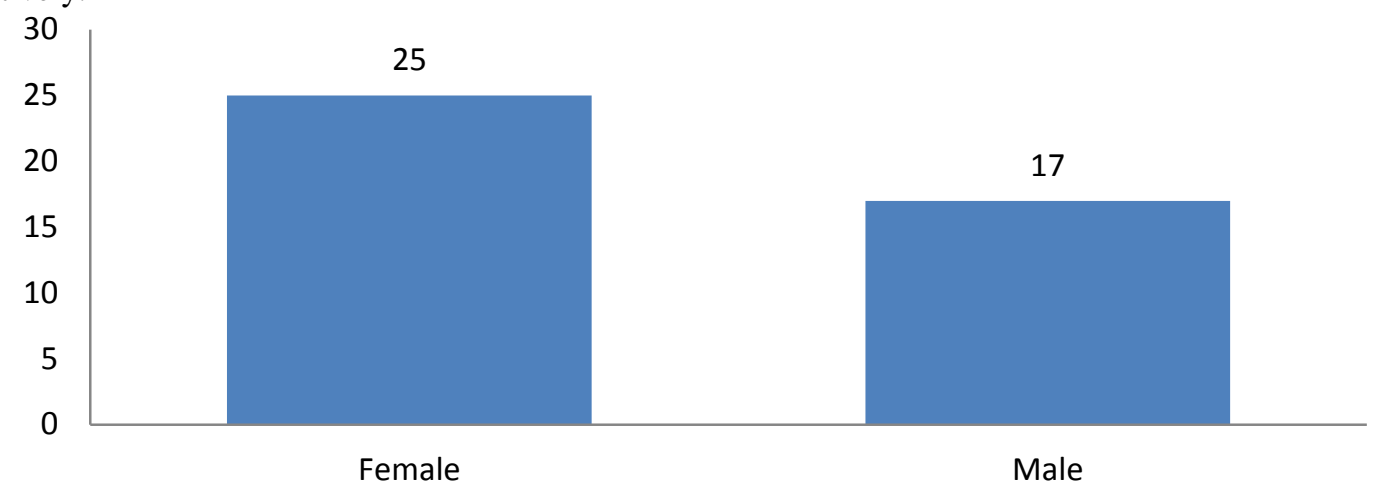

Fig. 3 above shows the occurence of Escherichia coli in the study population. Out of $42(51.2 \%)$ E. coli isolated from the overall study, $25(59.5 \%)$ were from females while $17(40.5 \%)$ were from males.

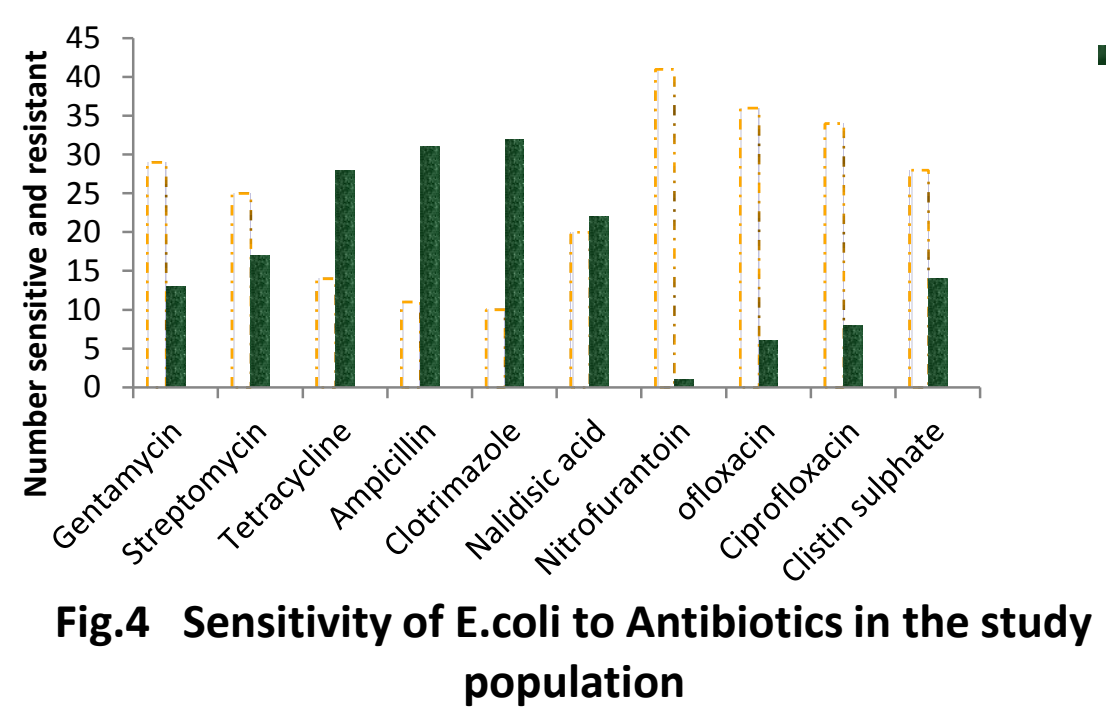

Fig. 4 shows the sensitivity of E. coli to the antibiotics in use in the hospital. E. coli exhibited greater sensitivity to Nitrofurantoin, accounting for 97.6\%. followed by Ofloxacin (85.7\%), Ciprofloxacin (80.9\%) and Gentamycin $(69 \%)$. On the other hand, $(76.1 \%)$ of the isolates showed greater resistance to Cotrimoxazole (76.1\%), Ampicillin (73.8\%), and Tetracycline (66.6\%).

\section{Discussion}

From the result, more patients turned out to be uninfected. This finding is an indication that, although these symptoms could suggest infection of the urinary tract, bacteriuria is not their exclusive cause. Patients with classic symptoms of urinary tract infection may have a sterile urine, while asymptomatic patients may have infected urine (Najar, et al, 2009). Investigators have found that only one-half of women with symptoms of acute lower UTI met the criterion of $\geq 10^{5}$ colony forming unit per milliliter (c.f.u./ml). Low-count bacteriuria might be an early phase of UTI. The majority of patients with bacterial counts between $10^{2}$ and $10^{4}$ c.f.u. $/ \mathrm{ml}$ has micro-organisms typical for UTI (E. coli, Staphylococcus saprophyticus, and enteric Gram-negative bacteria) (Kunin et al, 1993., Avar-Boger et al, 1994). Based on what was observed from the results obtained, women have higher percentage rate of infection than men. The urinary tract infection occur more frequently in women than in men. This differences could be attributed to several factors such as the anatomical differences between the male and female urethra, improper cleaning of the perineum, the use of napkins, sanitary towels and tampons together with pregnancy and intercourse contribute to the higher occurrence of the infection in various categories of women (Hooton et al, 1996 ). In addition, the urine of females was found to have a more suitable $\mathrm{PH}$ and osmolarity for the growth of Escherichia coli, and other enteric pathogens. 
Out of the five uropathogens (Escherichia coli Proteus species, Klebsiella species, Pseudomonas species and staphylococcus aureus) isolated during the period of the study, E.coli was the most common and this corresponds with the work done by Ozumba et al, (1993) who reported that E. coli is the commonest pathogen causing urinary tract infected accounting for $80.4 \%$ infection at first attack and $66.2 \%$ in second attack while cheesbrough, (2000) also confirmed that urinary tract infection is found more in women. But the most common, Escherichia coli is responsible for approximately $80 \%$ of the infection (Nidus information services, 2001) and from the result of the study population, E. coli were isolated from 59.5\% of infected females and $40.5 \%$ of infected males

E. coli. was tested against 10 different antimicrobial drugs namely gentamycin, streptomycin, ampicillin, cotrimazole, nalidixic acid, nitrofurantoin, ofloxacin, ciprofloxacin and colistin sulphate. Escherichia coli was found to be $97.6 \%$ sensitive to nitrofurantoin, $85.7 \%$ to ofloxacin, and $80.9 \%$ to ciprofloxacin, $69 \%$ to Gentamycin and $73.8 \%$ resistant to ampicillin, $66.6 \%$ to tetracycline and $76.1 \%$ to cotrimoxazole were detected. Sensitivity of E. coli. to nitrofurantoin, ofloxacin and ciprofloxacin are largely due to uncommon use of these drugs as opposed to the rampant use of ampicillin, tetracycline and cotrimoxazole where they grew resistant. The antibiotic sensitivity and resistance correlates with what was reported by Ozumba et. al 1993 and Adeleke et al 2005. The E. coli. resistance to ampicillin, tetracycline and cotrimoxazole is likely to be due to widespread indiscriminate abuse of these antibiotics in Zaria community, being easily available from patent medicine stores without doctor's prescription. Antibiotic sensitivity of bacteria depends to a large extent on the pattern of use of antibiotic in the particular environment. Owing to the prevalence of gastrointestinal, respiratory and other infections in the tropics, the same antibiotics are used for urinary tract infection. The antibiotic sensitivity obtainable in this community, Zaria could be different from that in developed countries (Gruneberg, 1980., Saffar et al., 2008). In developed countries, most primary urinary infection would respond to a course of sulphonamide. In many developing countries where laboratory facilities are limited even in hospital, it is important to know the antibiotic sensitivity of the organism for effective primary treatment.

\section{Conclusion}

Among the 10 antibiotics used for the sensitivity test in this study, nitrofurantoin, ciprofloxacin, ofloxacin and gentamycin demonstrated good therapeutic agents against urinary tract infection caused by Escherichia coli in Zaria community and should be used cautiously to avoid resistance from them too.

\section{References}

[1] S.I. Adeleke, M.O. Asani, R.O. Belonwu, G.U. Ihesiulor. Urinary tract pathogens and antimicrobial sensitivity patterns in childhood urinary infection, Kano, Nigeria. Ann. Nig. Med., 1(2): 2005, 14-16.

[2] B. Arav-Boger, L. Leibovici, Y.L. Danon. Urinary tract infections with low and high colony counts in young women. Spontaneous remission and single-dose vs multiple-day treatment. Arch Intern Med 1994; 154: 300-304

[3] M. Cheesbrough, District Laboratory Practice in tropical countries. 2000. Part 2.159-162

[4] L.G. Feld, T.K. Mattoo. Urinary Tract Infections and Vesicoureteral Reflux in Infants and Children. Pediatr Rev. (11); 2010. 45163

[5] R.N. Gruneberg Antibiotic sensitivities of urinary pathogens 1971-1978. J. Clin. Pathol., 33: 1980. 853-856

[6] Hooton TM, Scholes D, Hughes JP, Winter C, Roberts PL, Stapleton AE, et al. A prospective study of risk factors for symptomatic urinary tract infections in young women. N Engl J Med. 1996;335:468-74.

[7] H. Inabo H. Obanibi. Antimicrobial susceptibility of some urinary tract clinical isolates to commonly used antibiotics. Afr $J$ Biotechnol.5(5): 2006. 487-9.

[8] B. John. and L.L Mecheal. Urinary Tract Infection During Pregnancy. (Handout) University of Missouri - Columbia School of Medicine. 2000.( 721)

[9] C.M Kunin, L.V. White, H.H. Tong. A reassessment of the importance of 'low-count' bacteriuria in young women with acute urinary symptoms. Ann Intern Med 1993; 119: 454-460

[10] M. S. Najar, C. L. Saldanha and K. A. Banday. Approach to urinary tract infections Indian J

Nephrol. 2009 October; 19(4): 129-139.

[11] Nicolle LE Complicated urinary tract infection in adults. Can J Infect Disease and Medical Microbiol. 16(6): 2005. 349-360

[12] Nidus Information services Inc. Urinary Tract Infection, Infectious agent symptoms. Risk factors Severity, Diagnosis, Antibiotic treatment other preventive measures, support organization. 2001.

[13] U.O. Ozumba, Bacteriology and Antibiotic Sensitivity in acute Urinary Tract Infections in Enugu, Orient Journal med. 5 (2\&3). 1993, 69,

[14] S. Paul. Bacteria in Biology/Biotechnology and Medicine 4th Edition, (Wiley, John \& sons incorporated) 1997

[15] R. Ranjbar. M. Haghi-Ashtiani, N.J. Jafari, M. Abedini. The prevalence and antimicrobial susceptibility of bacterial uropathogens isolated from pediatric patients. Iranian J Pub Health. 2009: 321-6.

[16] Richard, J. Wagman, M.D.FACP (1983): Medical Health Encylopidia.

[17] M.J. Saffar, A.A. Enayti, I.A. Abdolla, M.S. Razai, H. Saffar H (2008). Antibacterial susceptibility of uropathogens in 3 hospitals, Sari, Iran, 2002-2003. Eastern Mediterranean Health Journal., 2008. 14.

[18] B.E. Scully, Pharmacology of the Floroquinalones. Supplement Urology 35(1):1990, 8-10 